\title{
High-Normal Thyroid Function and Risk of Recurrence of Atrial Fibrillation After Catheter Ablation
}

\author{
Ri-Bo Tang, MD; Dong-Ling Liu, MD; Jian-Zeng Dong, MD; Xing-Peng Liu, MD; \\ De-Yong Long, MD; Rong-Hui Yu, MD; Fu-Li Hu, MD; Jia-Hui Wu, MD; \\ Xiao-Hui Liu, MD; Chang-Sheng Ma, MD
}

\begin{abstract}
Background: It has been shown that the concentration of serum free thyroxine $\left(\mathrm{FT}_{4}\right)$ is independently associated with atrial fibrillation (AF), even in euthyroid persons. This study investigated the effect of a high-normal level of $\mathrm{FT}_{4}$ on recurrence after catheter ablation of $\mathrm{AF}$.

Methods and Results: The 244 consecutive patients with paroxysmal AF and who underwent circumferential pulmonary vein isolation (PVI) were prospectively enrolled. Exclusion criteria included prior or current thyroid dysfunction on admission, amiodarone medication for 3 months before admission. After a mean follow-up of $416 \pm 204$ (91-856) days, the recurrence rates were $14.8 \%, 23.0 \%, 33.3 \%, 38.7 \%$ from the lowest $\mathrm{FT}_{4}$ quartile to the highest $\mathrm{FT}_{4}$ quartile, respectively $(\mathrm{P}=0.016)$. After adjustment for age, sex, left atrial diameter, and $\mathrm{PVI}$, there was an increased risk of recurrence in the subjects with the highest $\mathrm{FT}_{4}$ quartile compared with those with the lowest quartile (hazard ratio 3.31, 95\% confidence interval 1.45-7.54, $\mathrm{P}=0.004$ ). As a continuous variable, $\mathrm{FT}_{4}$ was also an independent predictor of recurrence (hazard ratio 1.10, 95\% confidence interval 1.02-1.18, $P=0.016$ ).
\end{abstract}

Conclusions: Patients with high-normal thyroid function were at an increased risk of AF recurrence after catheter ablation. (Circ J 2010; 74: 1316-1321)

Key Words: Atrial fibrillation; Catheter ablation; Recurrence; Thyroid

$\mathbf{T}$ hyroid dysfunction, especially hyperthyroidism, is frequently associated with the development of atrial fibrillation $\mathrm{AF}$ ) and the prevalence of $\mathrm{AF}$ in patients with hyperthyroidism is significantly higher than that in euthyroid subjects. ${ }^{1-3}$ A previous study reported that the incidence of AF was $2.3 \%$ in patients with normal levels of serum thyroid-stimulating hormone (TSH), but increased to $13.8 \%$ in patients with hyperthyroidism. ${ }^{1}$ Even in patients with subclinical hyperthyroidism, a 5-fold increase in the risk of $\mathrm{AF}$ was observed. ${ }^{1}$ According to the recent guideline, restoring to euthyroid and rate control were recommended for patients with hyperthyroidism-related $\mathrm{AF}^{4}{ }^{4}$ In our previous study of patients with hyperthyroidism-related $\mathrm{AF}$ who had been euthyroid for more than 3 months, catheter ablation of AF guided by a 3-D mapping system was a therapeutic option. ${ }^{5}$ However, the impact of thyroid function on the recurrence rate was not determined according to thyroid function stratification was a limitation of the study. A crosssectional population-based study showed that the serum free thyroxine $\left(\mathrm{FT}_{4}\right)$ concentration was independently associated with AF, even in euthyroid persons with TSH levels in the normal range. ${ }^{3}$ Because most of the AF pathogenic factors present in patients with high levels of $\mathrm{FT}_{4}$ remain even after ablation, it is reasonable to hypothesize that $\mathrm{FT}_{4}$ at a highnormal level increases the risk of recurrence of AF after catheter ablation, so we conducted a prospective study to test this hypothesis.

\section{Methods \\ Study Subjects \\ Consecutive patients with refractory symptomatic paroxys- mal AF who were hospitalized in Beijing An Zhen Hospital (affiliated to Capital Medical University) for index circum- ferential pulmonary vein $(\mathrm{PV})$ radiofrequency ablation from January 2006 to November 2007 were prospectively enrolled. Exclusion criteria included thyroid dysfunction on admis- sion (abnormal level of FT4 or TSH), prior thyroid dysfunc-}

Received October 8, 2009; revised manuscript received February 26, 2010; accepted March 8, 2010; released online May 27, 2010 Time for primary review: 18 days

Department of Cardiology (R.-B.T., D.-L.L., J.-Z.D., X.-P.L., D.-Y.L., R.-H.Y., J.-H.W., X.-H.L., C.-S.M.), Beijing An Zhen Hospital, Capital Medical University, Beijing Institute of Heart Lung and Blood Vessel Diseases, Beijing; Department of Cardiology (F.-L.H.), Hebei General Hospital, Shijiazhuang, China

The first two authors contributed equally to the study (R.-B.T., D.-L.L.).

Mailing address: Chang-Sheng Ma, MD, Department of Cardiology, Beijing An Zhen Hospital affiliated to Capital Medical University, Chaoyang District, Beijing 100029, PR China. E-mail: chshma@vip.sina.com

ISSN-1346-9843 doi:10.1253/circj.CJ-09-0708

All rights are reserved to the Japanese Circulation Society. For permissions, please e-mail: cj@j-circ.or.jp 


\begin{tabular}{|c|c|c|c|c|c|}
\hline & \multicolumn{4}{|c|}{$\mathrm{FT}_{4}$ level (pmol/L) } & \multirow[b]{2}{*}{$P$ value } \\
\hline & $\begin{array}{c}Q_{1} \\
(<13.4) \\
(n=61)\end{array}$ & $\begin{array}{c}Q_{2} \\
(13.4-15.2) \\
(n=61)\end{array}$ & $\begin{array}{c}Q_{3} \\
(15.2-18.4) \\
(n=60)\end{array}$ & $\begin{array}{c}Q_{4} \\
(>18.4) \\
(n=62)\end{array}$ & \\
\hline Age (years) & $54 \pm 13$ & $58 \pm 11$ & $56 \pm 13$ & $56 \pm 12$ & 0.250 \\
\hline Male, n (\%) & $39(63.9 \%)$ & $39(63.9 \%)$ & $47(78.3 \%)$ & $52(83.9 \%)$ & $0.023^{*}$ \\
\hline AF duration (years) & $6.0 \pm 5.2$ & $7.1 \pm 5.7$ & $5.7 \pm 7.3$ & $6.0 \pm 5.7$ & 0.559 \\
\hline Heart rate (beats/min) & $73 \pm 11$ & $71 \pm 10$ & $71 \pm 11$ & $73 \pm 14$ & 0.642 \\
\hline Hypertension, n (\%) & $23(37.7 \%)$ & $33(54.1 \%)$ & $26(43.3 \%)$ & $28(45.2 \%)$ & 0.332 \\
\hline Structural heart disease, n (\%) & $6(9.8 \%)$ & $6(9.8 \%)$ & $7(11.7 \%)$ & $9(14.5 \%)$ & 0.828 \\
\hline Diabetes mellitus, n (\%) & $4(11.4 \%)$ & $5(14.7 \%)$ & $2(8.0 \%)$ & $5(17.9 \%)$ & 0.732 \\
\hline Left atrial diameter (mm) & $36.0 \pm 5.6$ & $36.5 \pm 5.3$ & $37.7 \pm 6.3$ & $39.8 \pm 6.8$ & $0.003^{*}$ \\
\hline LVESD (mm) & $30.9 \pm 4.9$ & $31.0 \pm 4.2$ & $32.5 \pm 6.7$ & $33.0 \pm 6.8$ & 0.126 \\
\hline LVEDD (mm) & $48.2 \pm 4.7$ & $48.0 \pm 5.0$ & $48.9 \pm 5.6$ & $48.4 \pm 10.2$ & 0.880 \\
\hline Ejection fraction (\%) & $64.6 \pm 6.7$ & $64.7 \pm 5.7$ & $62.7 \pm 8.2$ & $62.5 \pm 9.4$ & 0.245 \\
\hline$\beta$-blockers, $\mathrm{n}(\%)$ & $9(14.8 \%)$ & $15(24.6 \%)$ & $9(15.0 \%)$ & $14(22.6 \%)$ & 0.384 \\
\hline ACEI/ARB, $n(\%)$ & $20(32.8 \%)$ & $24(39.3 \%)$ & $19(31.7 \%)$ & $21(33.9 \%)$ & 0.816 \\
\hline Statins, n (\%) & $5(8.2 \%)$ & $11(18.0 \%)$ & $6(10.0 \%)$ & $12(19.4 \%)$ & 0.185 \\
\hline TSH (mIU/L) & $2.270 \pm 1.063$ & $1.848 \pm 0.955$ & $1.868 \pm 1.074$ & $1.981 \pm 0.906$ & 0.078 \\
\hline Pulmonary vein isolation, $\mathrm{n}(\%)$ & $58(95.1 \%)$ & $60(98.4 \%)$ & $55(91.7 \%)$ & $62(100 \%)$ & 0.072 \\
\hline Procedure time (min) & $164 \pm 42$ & $171 \pm 39$ & $172 \pm 40$ & $174 \pm 40$ & 0.534 \\
\hline Fluoroscopy time (min) & $32 \pm 8$ & $34 \pm 8$ & $34 \pm 8$ & $35 \pm 9$ & 0.348 \\
\hline
\end{tabular}

*Statistically significant value $(P<0.05)$.

$\mathrm{FT}_{4}$, free thyroxine; $\mathrm{AF}$, atrial fibrillation; LVESD, left ventricular end-systolic diameter; LVEDD, left ventricular end-diastolic diameter; ACEI, angiotensin-converting enzyme inhibitors; ARB, angiotensin-receptor blockers; TSH, thyroid-stimulating hormone.

tion, and patients who had been on amiodarone for 3 months before admission. In total, 244 patients were enrolled and 112 patients were excluded. All patients gave written informed consent, and the study was approved by the institutional review board.

Blood samples were collected after overnight fasting on the first morning of hospitalization. Serum TSH FT 4 and $\mathrm{FT}_{3}$ levels were assessed with a commercial assay (Vitros ECi Immunodiagnostic System; Ortho-Clinical Diagnostics, USA). The laboratory reference range of $\mathrm{FT}_{4}$ was $10.0-28.2 \mathrm{pmol} / \mathrm{L}$, with an interassay coefficient of variation of 4.4-5.8\% (range, $6.36-47.5 \mathrm{pmol} / \mathrm{L}$ ). The laboratory reference range of $\mathrm{FT}_{3}$ was 3.48-9.46 pmol/L, with an interassay coefficient of variation of $4.5-9.6 \%$ (range $3.60-21.7 \mathrm{pmol} / \mathrm{L}$ ). The reference range of serum TSH concentration was $0.46-4.68 \mathrm{mIU} / \mathrm{L}$, with an interassay coefficient of variation of 4.6-8.0\% (range $0.16-39.0 \mathrm{mIU} / \mathrm{L}$ ). The lowest limit value of $\mathrm{FT}_{4}$ assay reported was $0.700 \mathrm{pmol} / \mathrm{L}$, with a mean functional sensitivity $0.40 \mathrm{pmol} / \mathrm{L}$. Transesophageal and transthoracic echocardiography images were obtained prior to ablation in order to measure the left atrial (LA) anteroposterior diameter, left ventricular (LV) end-diastolic diameter, LV end-systolic diameter, and LV ejection fraction, and to exclude the presence of intra-atrial thrombus.

\section{Electrophysiological Study and Follow-up}

All 244 patients in this study underwent circumferential PV ablation. The ablation procedure was performed in the postabsorptive state. The technique of circumferential PV ablation guided by 3-D LA mapping has been previously described in detail. ${ }^{6,7}$ Briefly, the LA was explored by a transseptal approach. The LA geometry was reconstructed with a $3.5 \mathrm{~mm}$ tip ablation catheter (Navi-Star ThermoCool, BiosenseWebster, USA) in the CARTO system. A continuous irrigated radiofrequency ablation was performed along each PV antrum in order to encircle the ipsilateral PVs (target temperature: $45^{\circ} \mathrm{C}$, maximum power: $35 \mathrm{~W}$, infusion rate: $17 \mathrm{ml} / \mathrm{min}$ ). Procedural endpoints were completeness of continuous circular lesions and electrical isolation of all PVs. If a typical atrial flutter had been documented before the procedure, the tricuspid isthmus responsible for this tachycardia was identified and ablated. Bidirectional conduction block was achieved if the tricuspid isthmus was targeted.

After the procedure, amiodarone was administered to the patient if there were neither contraindications nor intolerance. If no recurrent atrial tachyarrhythmia occurred after 2 or 3 months, amiodarone was discontinued. All asymptomatic patients were followed up with 12-lead ECG and 24-h Holter recordings before discharge and at $1,3,6$ and 12 months after the ablative procedure. If the patient was symptomatic, a new ECG was obtained. In addition, a physician conducted monthly telephone interviews of all patients. Recurrence was defined as the occurrence of confirmed atrial tachyarrhythmia (documented by ECG or Holter recordings) beyond 3 months after the index catheter ablation while off antiarrhythmic medication.

\section{Statistical Analysis}

Continuous data are presented as mean \pm standard deviation and were compared by Student's t-test or 1-way ANOVA analysis. If variables were not in normal distribution, the Mann-Whitney U test was performed. Categorical variables were compared by chi-square analysis. Serum FT4 level was categorized into quartiles and a Kaplan-Meier estimation with a log-rank test served for the unadjusted analysis of the effect of $\mathrm{FT}_{4}$ on the recurrence of catheter ablation of AF. A Cox proportional hazards model was used to estimate the relationship between $\mathrm{FT}_{4}$ and the recurrence of AF. Age, sex, LA diameter, and PV isolation (PVI) were adjusted in the Cox analysis. All analyses were performed with SPSS 


\begin{tabular}{|c|c|c|c|}
\hline & $\begin{array}{l}\text { Non-recurrence } \\
\quad(n=177)\end{array}$ & $\begin{array}{l}\text { Recurrence } \\
(n=67)\end{array}$ & $P$ value \\
\hline Age (years) & $56 \pm 12$ & $54 \pm 12$ & 0.283 \\
\hline Male, n (\%) & $123(69.5 \%)$ & $54(80.6 \%)$ & 0.083 \\
\hline AF duration (years) & $6.4 \pm 6.3$ & $5.8 \pm 5.2$ & 0.476 \\
\hline Hypertension, n (\%) & $84(47.5 \%)$ & $26(38.8 \%)$ & 0.225 \\
\hline Structural heart disease, $\mathrm{n}(\%)$ & $22(12.4 \%)$ & $6(9.0 \%)$ & 0.447 \\
\hline Diabetes mellitus, n (\%) & $11(12.0 \%)$ & $5(16.7 \%)$ & 0.507 \\
\hline Left atrial diameter (mm) & $37.3 \pm 5.9$ & $38.1 \pm 6.9$ & 0.387 \\
\hline LVEDD (mm) & $48.2 \pm 7.2$ & $48.8 \pm 5.2$ & 0.585 \\
\hline LVESD (mm) & $31.8 \pm 6.0$ & $32.1 \pm 5.3$ & 0.693 \\
\hline Ejection fraction (\%) & $64.1 \pm 7.4$ & $62.4 \pm 8.2$ & 0.131 \\
\hline$\beta$-receptor blockers, $\mathrm{n}(\%)$ & $31(17.5 \%)$ & $16(23.9 \%)$ & 0.260 \\
\hline ACEI/ARB, $n(\%)$ & $63(35.5 \%)$ & $21(31.4 \%)$ & 0.533 \\
\hline Statins, n (\%) & $26(14.7 \%)$ & $8(11.9 \%)$ & 0.580 \\
\hline TSH (mIU/L) & $2.009 \pm 0.988$ & $1.947 \pm 1.069$ & 0.670 \\
\hline $\mathrm{FT}_{4}(\mathrm{pmol} / \mathrm{L})$ & $15.6 \pm 3.3$ & $16.8 \pm 3.2$ & $0.013^{*}$ \\
\hline $\mathrm{FT}_{4}$ quartile (pmol/L) & & & $0.014^{*}$ \\
\hline$Q_{1}(<13.4)$ & $52(29.4 \%)$ & $9(13.4 \%)$ & \\
\hline$Q_{2}(13.4-15.2)$ & $47(26.6 \%)$ & $14(20.9 \%)$ & \\
\hline$Q_{3}(15.2-18.4)$ & $40(22.6 \%)$ & $20(29.9 \%)$ & \\
\hline $\mathrm{Q}_{4}(>18.4)$ & $38(21.5 \%)$ & $24(35.8 \%)$ & \\
\hline Pulmonary vein isolation, $\mathrm{n}(\%)$ & $172(97.2 \%)$ & $63(94.0 \%)$ & 0.245 \\
\hline
\end{tabular}

*Statistically significant value $(\mathrm{P}<0.05)$. Abbreviations as in Table 1.

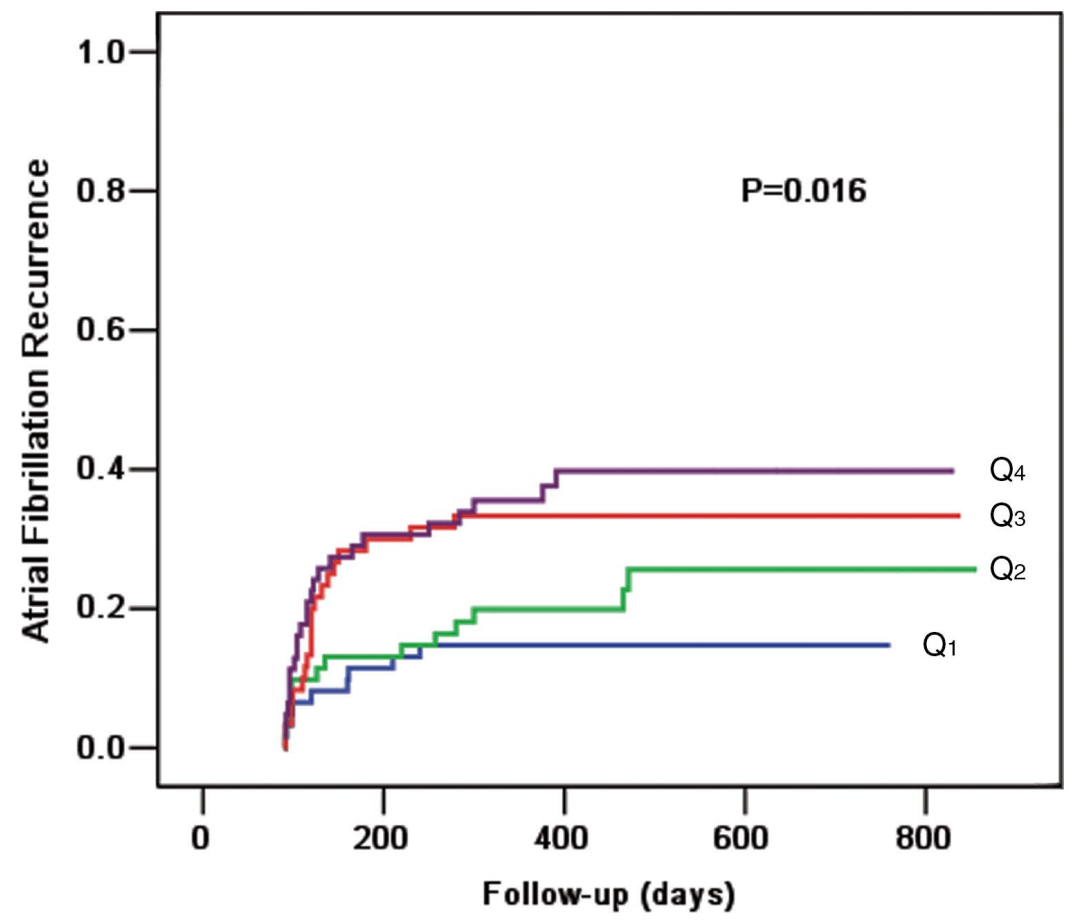

Figure. Kaplan-Meier curves depicted recurrence of atrial fibrillation in patients with different quartiles of serum free thyroxine. From the lowest quartile $\left(Q_{1}\right)$ to the highest quartile $\left(\mathrm{Q}_{4}\right)$, the recurrence rates were $14.8 \%, 23.0 \%, 33.3 \%$, $38.7 \%$, respectively $(P=0.016)$.

software version 13.0. All probability values were 2-sided, and $\mathrm{P}<0.05$ was considered statistically significant.

\section{Results}

The patient characteristics according to $\mathrm{FT}_{4}$ quartile are listed in Table 1. From the lowest to the highest $\mathrm{FT}_{4}$ quartile, the LA diameter was $36.0 \pm 5.6 \mathrm{~mm}, 36.5 \pm 5.3 \mathrm{~mm}, 37.7 \pm 6.3 \mathrm{~mm}$, $39.8 \pm 6.8 \mathrm{~mm}$, respectively $(\mathrm{P}=0.006)$. Statistically significant differences of sex were found among the quartiles. There were no differences in age, AF duration, heart rate prior to the procedure, LV end-diastolic diameter, LV end-systolic 


\begin{tabular}{|c|c|c|c|c|}
\hline & \multicolumn{2}{|c|}{ Model 1} & \multicolumn{2}{|c|}{ Model 2} \\
\hline & $P$ value & HR $(95 \% \mathrm{Cl})$ & $P$ value & HR $(95 \% \mathrm{Cl})$ \\
\hline Age & 0.480 & $0.99(0.97-1.01)$ & 0.555 & $0.99(0.97-1.01)$ \\
\hline Male & 0.468 & $1.28(0.66-2.48)$ & 0.370 & $1.35(0.70-2.62)$ \\
\hline Left atrial diameter & 0.724 & $1.01(0.96-1.05)$ & 0.758 & $1.01(0.96-1.05)$ \\
\hline Pulmonary vein isolation & 0.277 & $0.55(0.19-1.61)$ & 0.245 & $0.54(0.19-1.52)$ \\
\hline $\mathrm{FT}_{4}(\mathrm{pmol} / \mathrm{L})$ & - & - & $0.016^{*}$ & $1.10(1.02-1.18)$ \\
\hline$Q_{1}(<13.4)$ & & Reference & & \\
\hline $\mathrm{Q}_{2}(13.4-15.2)$ & 0.194 & $1.79(0.74-4.29)$ & & \\
\hline $\mathrm{Q}_{3}(15.2-18.4)$ & 0.063 & $2.24(0.96-5.24)$ & & \\
\hline $\mathrm{Q}_{4}(>18.4)$ & $0.004^{*}$ & $3.31(1.45-7.54)$ & & \\
\hline
\end{tabular}

*Statistically significant value $(P<0.05)$.

$\mathrm{HR}$, hazard ratio; $\mathrm{Cl}$, confidence interval. Other abbreviations as in Table 1.

diameter, or LV ejection fraction among the $\mathrm{FT}_{4}$ quartiles. The prevalence of diabetes mellitus, hypertension, structural heart disease (including valvular heart disease, dilated, hypertrophic or ischemic cardiomyopathy, heart failure, coronary artery disease), medications (angiotensin-converting enzyme inhibitors/angiotensin receptor blockers, statins, $\beta$-blockers) did not differ significantly among the quartiles. There were no significant differences in procedure time, fluoroscopy time, or the PVI rate among the quartiles.

After a mean follow-up of 416 \pm 204 (91-856) days, 67 patients $(26.2 \%)$ experienced recurrence. The characteristics of the recurrence and non-recurrence groups are shown in Table 2. The level of serum $\mathrm{FT}_{4}$ was significantly higher in the recurrence group than in the non-recurrence group (16.8 \pm $3.2 \mathrm{pmol} / \mathrm{L}$ vs $15.6 \pm 3.3 \mathrm{pmol} / \mathrm{L}, \mathrm{P}=0.006)$. There was significant difference in the prevalence of $\mathrm{FT}_{4}$ quartiles between the recurrence group and the non-recurrence group. As it was shown in Figure, from the lowest $\mathrm{FT}_{4}$ quartile to the highest quartile, the recurrence rates were $14.8 \%, 23.0 \%, 33.3 \%$, $38.7 \%$, respectively $(\mathrm{P}=0.016)$. There was no significant difference in the $\mathrm{FT}_{3}$ level between the recurrence and nonrecurrence groups $(5.75 \pm 0.59 \mathrm{pmol} / \mathrm{L}$ vs $5.91 \pm 0.83 \mathrm{pmol} / \mathrm{L}$, $\mathrm{P}=0.144)$. The recurrence rates were $23.0 \%, 28.3 \%, 24.2 \%$, $34.4 \%$ from the lowest to the highest $\mathrm{FT}_{3}$ quartile, respectively $(\mathrm{P}=0.114)$. The LA diameter and PVI rate did not differ between the 2 groups. In total, 31 patients underwent redo procedures, and 93.5\% (29/31) had PV reconnection, but the PV reconnection rate did not differ among the $\mathrm{FT}_{4}$ quartiles (8/8 [100\%], 3/4 [75\%], 11/11 [100\%], 7/8 [87.5\%], $\mathrm{P}=0.253$ ).

Considering $\mathrm{FT}_{4}$ as a category variate in model 1 (Table 3), after adjustment for age, sex, LA diameter, and PVI, there were an increased risk of recurrence in subjects in the highest quartile of $\mathrm{FT}_{4}$ after catheter ablation of AF compared with subjects in the lowest quartile (hazard ratio [HR] 3.31, 95\% confidence interval $[\mathrm{CI}] 1.45-7.54, \mathrm{P}=0.004)$. Considering $\mathrm{FT}_{4}$ as a continuous variate in model 2 (Table 3 ), after adjustment for age, sex, LA diameter, and PVI, Cox analysis revealed that $\mathrm{FT}_{4}$ was also an independent predictor of recurrence (HR 1.10, 95\%CI 1.02-1.18, $\mathrm{P}=0.016)$.

\section{Discussion}

The main finding of the present study is that a high-normal level of thyroid function is an independent predictor of recurrence of AF after catheter ablation of paroxysmal AF.

Hyperthyroidism is an important factor in the etiology of paroxysmal AF. ${ }^{3,8,9}$ Not only overt hyperthyroidism, a wellknown risk factor for AF, ${ }^{1,3,10}$ but also subclinical hyperthyroidism, which is defined as a low TSH level with a serum $\mathrm{FT}_{4}$ concentration within the normal range. 1,10,11 $^{\text {Thyroid hor- }}$ mone has some effects on cardiovascular function. Previous studies have shown that thyroid hormone can shorten the action potential of both atrial and ventricular myocytes, which might facilitate the occurrence of reentry and circuits and contribute to the high incidence of atrial tachyarrhythmias in patients with hyperthyroidism. ${ }^{12,13}$ It was reported that the PV play an important role in the onset of paroxysmal AF. ${ }^{14,15}$ Chen et al found that hyperthyroid atrial myocytes and PVs had a short action potential duration. ${ }^{16}$ Hyperthyroid PV cardiomyocytes might cause faster beating rates, a higher incidence of delay after depolarization, and a higher incidence of early afterdepolarization. ${ }^{16}$ Therefore, increased automaticity and enhanced triggered activity may increase the arrhythmogenic activity of the PV in hyperthyroidism. ${ }^{16,17}$

Thyroid hormone changes the electrophysiology of atrial myocyte and PV. The arrhythmogenic effect of thyroid hormone might exist even in euthyroid subjects. In a populationbased study, higher $\mathrm{FT}_{4}$ concentrations were found in those with AF compared with those without AF in a subgroup analysis confined to 5,519 euthyroid subjects. ${ }^{3}$ In logistic analysis, serum $\mathrm{FT}_{4}$ was an independent predictor of AF. In the Rotterdam study, the multivariate adjusted level of $\mathrm{FT}_{4}$ showed a graded association with the risk of new onset AF. ${ }^{18}$

The aforementioned findings supported the hypothesis that within the normal range of thyroid function, higher thyroid function confers a higher risk of AF. It was reasonable to believe that a high level of FT4 would lower the success rate of catheter ablation of AF. In this study, we sought to address the outcome of catheter ablation of $\mathrm{AF}$ in a subgroup of patients. To the best of our knowledge, our data show for the first time that a high-normal level of $\mathrm{FT}_{4}$ increases the incidence of recurrence after catheter ablation of paroxysmal AF in both univariate and multivariate analysis. Several potential mechanisms could cause this effect of $\mathrm{FT}_{4}$ on recurrence of $\mathrm{AF}$, including elevation of LA pressure secondary to increased LV mass and impaired ventricular relaxation, ${ }^{17}$ enhanced automaticity and triggered activity in PV cardiomyocytes, ${ }^{16}$ ischemia resulting from raised resting heart rate, and increased atrial ectopic activity. ${ }^{19}$ According to the study by Fazio et al, ${ }^{17}$ increased LA pressure should be a mechanism of increasing the LA diameter. The present study also showed that patients in the highest quartile of $\mathrm{FT}_{4}$ had the largest LA diameter. The LA diameter is a well-known pre- 
dictor of recurrence after catheter ablation of AF. ${ }^{20,21}$ However, after adjusting for LA size, a high-normal level of FT4 became an independent predictor of recurrence. FT 4 concentrations might be themselves modulated by "nonthyroidal" factors such as illnesses and drug therapies, leading to a reduction in the $\mathrm{FT}_{4}$ concentration, and the same factors are likely to increase the risk of AF. However, in this study the highest quartile of $\mathrm{FT}_{4}$ favored recurrence.

Amiodarone can potentially induce thyroid dysfunction. In a previous study, hypothyroidism occurred in up to $20 \%$ of patients taking amiodarone, and hyperthyroidism occurred in $3 \%$ of patients with sufficient dietary iodine. ${ }^{22}$ To avoid these effects of amiodarone, patients who had been taking amiodarone for 3 months prior to admission were excluded. What's more, antiarrhythmic drugs, especially amiodarone, did not lower the recurrence of AF in Turco et al's study. ${ }^{23}$

\section{Study Limitations}

The exact mechanism of higher AF recurrence rates after catheter ablation in patients with a high-normal level of $\mathrm{FT}_{4}$ remains to be investigated. PVI reconnection is a known key cause of AF recurrence in most studies. ${ }^{24,25}$ The PVI rate did not differ among the quartile of $\mathrm{FT}_{4}$ levels, but whether elevated $\mathrm{FT}_{4}$ could increase the likelihood of PV reconnection was not identified in this study. The atrial myocytes of the AF substrate remain after circumferential PV ablation in patients with a high-normal level of $\mathrm{FT}_{4}$, which might contribute to the higher recurrence rate. However, data on non-PV foci were not available in this study. According to Fazio et al, ${ }^{17} \mathrm{a}$ large LA diameter caused by increased LA pressure might increase the recurrence of $\mathrm{AF}$, but multivariate analysis in our study did not show a relation between large LA and recurrence of AF. It is also a limitation that the diagnosis of $\mathrm{AF}$ recurrence was based on symptoms, ECG and Holter-ECG findings. Potentially, the AF recurrence rate might be underestimated because of asymptomatic cases. However, most of the patients studied were symptomatic with paroxysmal AF. Asymptomatic recurrence of AF after an apparently successful catheter ablation procedure of symptomatic paroxysmal $\mathrm{AF}$ was infrequent in a study that used transtelephonic event recorders for the follow-up. ${ }^{26}$ The sample size of the present study was not large enough, with approximately 60 patients in each quartile, which may have caused a statistical bias. A large study needs to be carried out in the future to explore the effect of thyroid hormone on the outcome of AF catheter ablation. Finally, how to prevent the recurrence of AF in patients with high-normal $\mathrm{FT}_{4}$ in clinical practice needs to be explored.

\section{Conclusion}

To the best of our knowledge, this study provides the first evidence that a high-normal level of $\mathrm{FT}_{4}$ is an independent predictor of AF recurrence after catheter ablation.

\section{Acknowledgments}

We thank Chang Liu for collecting the data and Jian-Jun Li for revising the manuscript. This work was supported by the National Science Foundation Council of China (No.30900628 and No.30670843), Beijing Natural Science Foundation (No.7102048) and Beijing Nova Program (No. 2009B32)

\section{Disclosure}

Conflicts of interest: none.

\section{References}

1. Auer J, Scheibner P, Mische T, Langsteger W, Eber O, Eber B. Subclinical hyperthyroidism as a risk factor for atrial fibrillation. Am Heart J 2001; 142: 838-842.

2. Frost L, Vestergaard P, Mosekilde L. Hyperthyroidism and risk of atrial fibrillation or flutter: A population-based study. Arch Intern Med 2004; 164: 1675-1678.

3. Gammage MD, Parle JV, Holder RL, Roberts LM, Hobbs FD, Wilson S, et al. Association between serum free thyroxine concentration and atrial fibrillation. Arch Intern Med 2007; 167: 928-934.

4. Fuster V, Ryden LE, Cannom DS, Crijns HJ, Curtis AB, Ellenbogen KA, et al. ACC/AHA/ESC 2006 Guidelines for the Management of Patients with Atrial Fibrillation: A report of the American College of Cardiology/American Heart Association Task Force on Practice Guidelines and the European Society of Cardiology Committee for Practice Guidelines (Writing Committee to Revise the 2001 Guidelines for the Management of Patients With Atrial Fibrillation): Developed in collaboration with the European Heart Rhythm Association and the Heart Rhythm Society. Circulation 2006; 114: e257-e354.

5. Ma CS, Liu X, Hu FL, Dong JZ, Liu XP, Wang XH, et al. Catheter ablation of atrial fibrillation in patients with hyperthyroidism. J Interv Card Electrophysiol 2007; 18: 137-142.

6. Liu X, Dong J, Mavrakis HE, Hu F, Long D, Fang D, et al. Achievement of pulmonary vein isolation in patients undergoing circumferential pulmonary vein ablation: A randomized comparison between two different isolation approaches. J Cardiovasc Electrophysiol 2006; 17: $1263-1270$.

7. Tang RB, Dong JZ, Liu XP, Long DY, Yu RH, Kalifa J, et al. Metabolic syndrome and risk of recurrence of atrial fibrillation after catheter ablation. Circ J 2009; 73: 438-443.

8. Polikar R, Burger AG, Scherrer U, Nicod P. The thyroid and the heart. Circulation 1993; 87: 1435-1441.

9. Woeber KA. Thyrotoxicosis and the heart. N Engl J Med 1992; 327: 94-98.

10. Sawin CT, Geller A, Wolf PA, Belanger AJ, Baker E, Bacharach $\mathrm{P}$, et al. Low serum thyrotropin concentrations as a risk factor for atrial fibrillation in older persons. N Engl J Med 1994; 331: 1249 1252 .

11. Tenerz A, Forberg R, Jansson R. Is a more active attitude warranted in patients with subclinical thyrotoxicosis? J Intern Med 1990; 228: 229-233.

12. Johnson PN, Freedberg AS, Marshall JM. Action of thyroid hormone on the transmembrane potentials from sinoatrial node cells and atrial muscle cells in isolated atria of rabbits. Cardiology 1973; 58: $273-289$.

13. Sun ZQ, Ojamaa K, Coetzee WA, Artman M, Klein I. Effects of thyroid hormone on action potential and repolarizing currents in rat ventricular myocytes. Am J Physiol Endocrinol Metab 2000; 278: E302-E307.

14. Dixit S, Gerstenfeld EP, Ratcliffe SJ, Cooper JM, Russo AM, Kimmel SE, et al. Single procedure efficacy of isolating all versus arrhythmogenic pulmonary veins on long-term control of atrial fibrillation: A prospective randomized study. Heart Rhythm 2008; 5: $174-181$.

15. Haissaguerre M, Jais P, Shah DC, Takahashi A, Hocini M, Quiniou $\mathrm{G}$, et al. Spontaneous initiation of atrial fibrillation by ectopic beats originating in the pulmonary veins. N Engl J Med 1998; 339: 659666.

16. Chen YC, Chen SA, Chen YJ, Chang MS, Chan P, Lin CI. Effects of thyroid hormone on the arrhythmogenic activity of pulmonary vein cardiomyocytes. J Am Coll Cardiol 2002; 39: 366-372.

17. Fazio S, Palmieri EA, Lombardi G, Biondi B. Effects of thyroid hormone on the cardiovascular system. Recent Prog Horm Res 2004; 59: $31-50$.

18. Heeringa J, Hoogendoorn EH, van der Deure WM, Hofman A, Peeters RP, Hop WC, et al. High-normal thyroid function and risk of atrial fibrillation: The Rotterdam study. Arch Intern Med 2008; 168: $2219-2224$.

19. Sgarbi JA, Villaca FG, Garbeline B, Villar HE, Romaldini JH. The effects of early antithyroid therapy for endogenous subclinical hyperthyroidism in clinical and heart abnormalities. J Clin Endocrinol Metab 2003; 88: $1672-1677$.

20. Sauer WH, McKernan ML, Lin D, Gerstenfeld EP, Callans DJ, Marchlinski FE. Clinical predictors and outcomes associated with acute return of pulmonary vein conduction during pulmonary vein isolation for treatment of atrial fibrillation. Heart Rhythm 2006; 3: $1024-1028$.

21. Pappone C, Rosanio S, Augello G, Gallus G, Vicedomini G, 
Mazzone P, et al. Mortality, morbidity, and quality of life after circumferential pulmonary vein ablation for atrial fibrillation: Outcomes from a controlled nonrandomized long-term study. J Am Coll Cardiol 2003; 42: 185-197.

22. Zimetbaum P. Amiodarone for atrial fibrillation. $N$ Engl $J$ Med 2007; 356: 935-941.

23. Turco P, De Simone A, La Rocca V, Iuliano A, Capuano V, Astarita $\mathrm{C}$, et al. Antiarrhythmic drug therapy after radiofrequency catheter ablation in patients with atrial fibrillation. Pacing Clin Electrophysiol 2007; 30(Suppl 1): S112-S115.

24. Yamada T, Yoshida N, Murakami Y, Okada T, Yoshida Y, Muto
M, et al. Vagal modification can be a valid predictor of late recurrence of paroxysmal atrial fibrillation independent of the pulmonary vein isolation technique. Circ J 2009; 73: 1606-1611.

25. Noguchi H, Kumagai K, Yasuda T, Ogawa M, Tojo H, Saku K. Conduction recovery after pulmonary vein isolation for atrial fibrillation. Circ J 2005; 69: 65-68.

26. Oral H, Veerareddy S, Good E, Hall B, Cheung P, Tamirisa K, et al. Prevalence of asymptomatic recurrences of atrial fibrillation after successful radiofrequency catheter ablation. J Cardiovasc Electrophysiol 2004; 15: 920-924. 